\title{
A REDUCED ORDER METHOD FOR SIMULATION AND CONTROL OF FLUID FLOWS *
}

\author{
KAZUFUMI ITO ${ }^{\dagger}$ AND S.S. RAVINDRAN ${ }^{\dagger}$
}

\begin{abstract}
This article presents a reduced order method for simulation and control of viscous incompressible flows. The major advantage of this method over others such as finite element, finite difference or spectral method is that it has fewer unknowns. The feasibility of this method for flow control is demonstrated on two boundary control problems. The first one is a velocity tracking problem in cavity flow and the second one is a vorticity control problem in channel flows. Our formulation of the reduced order method applied to flow control problems lead to constrained minimization problem and solved by applying Newton like methods to the necessary conditions of optimality. Through our computational experiments we demonstrate the feasibility and applicability of the reduced order method for control and time dependent problems in fluid flow calculations.
\end{abstract}

Key words. reduced basis method, Navier-Stokes equations, finite element, optimal control.

AMS subject classifications. 93B40, 49M05, 76D05, 49K20, 65H10

\section{CONTENTS}

1. Introduction.

1.1 Choices of Reduced Basis Subspaces.

1.2 Reduced Basis Error.

2. The Reduced Basis Method for Navier-Stokes Flows.

2.1 Notations.

2.2 Variational Formulation.

2.3 The reduced Basis Method and the Reduced Order Model.

2.3.1 Case I: Steady State.

2.3.2 Case II: Time Dependent State.

3. Computations of the Reduced Order Model.

3.1 Stationary Driven Cavity Problem.

3.2 Unsteady Channel Problem.

4. Control of Reduced Order Model.

4.1 Control of Driven Cavity Flow.

4.2 Control of Channel Flows.

4.2.1 Case I: Backward-Facing-Step Channel Flow.

4.2.2 Case II: Forward-Facing-Step Channel Flow.

5. Conclusion.

\footnotetext{
* This work was supported in part by the Air Force Office of Scientific Research under grants AFOSR F49620-95-1-0437 and AFOSR F49620-95-1-0447.

$\dagger$ Center for Research in Scientific Computation, Department of Mathematics, North Carolina State University, Raleigh, NC 27695-8205 (kito@eos.ncsu.edu and ravi@eos.ncsu.edu).
} 
1. INTRODUCTION. Control problems that involve partial differential equations as state equations are formidable problems to solve in real time. One such situation arises in control of fluid dynamical systems in which the state equations are the Navier-Stokes equations, the geometry is often complex and the time interval involved is often very large. If one were to solve such problems using standard finite element or finite difference method the resulting system is prohibitively large.

We in this article discuss a reduction type method which overcomes this difficulty. This method hereafter we call reduced basis method uses functions as basis functions which are closely related to the problem that is being solved. This is in contrast to the traditional numerical methods such as finite difference method which uses grid functions as basis functions or finite elements method which uses piecewise polynomials for this purpose.

There are several approaches available for the selection of basis functions. One such approach is Taylor approach in which one uses solutions at a point along with their derivatives as basis functions. Another approach which we call Lagrange approach uses solutions of the problem at various parameter values as basis functions. Finally the Hermite approach is a hybrid of Lagrange and Taylor approaches.

Our goal here is first to test and validate the reduced basis method for fluid flow simulations. Then use the resulting reduced order model for control problems in fluid flows. We will investigate both steady and unsteady flows and demonstrate the feasibility of the reduced model for flow control by performing computations on cavity, backward-facing-step channel flow and forward-facing-step channel flow.

The reduced basis has been applied to structural mechanics problems with considerable success, see [1],[9]-[13]. Its use for high Reynolds number fluid flow calculation has also been shown, see [14].

1.1. Choices of Reduced Basis Subspaces. In order to illustrate the reduced basis method, we assume for ease in exposition that we are dealing with nonlinear dynamics about the stable equilibrium points. Consider the the parameterized stationary problem

$$
\mathcal{E}(y, \mu)=0 \quad \text { for } \mu \in \mathbb{R}, y \in X,
$$

where $\mu$ represents some physical parameter, for example, Reynolds number or viscosity, about which we choose to interpolate to obtain a reduced finite dimensional set of basis elements. In standard finite element approximations, one approximates $\mathrm{X}$ with a piecewise polynomial space. However, the choices for the reduced basis method are different.

The Taylor Subspace. In this choice, one assumes at some value of $\mu$, say $\mu^{*}$, the solution is known and it has $\mathrm{M}$ derivatives then the reduced basis subspace $X_{R}$ is defined as

$$
X_{R}=\operatorname{span}\left\{y_{j}\left|y_{j}=\frac{\partial^{j} y}{\partial \mu^{j}}\right|_{\mu=\mu^{*}}, j=0, \ldots, M\right\},
$$

where $y^{j}$ is obtained from successive differentiation of (1.1), i.e.

$$
\mathcal{E}_{y}\left(y_{0}, \mu_{0}\right) y_{j}=\mathcal{F}_{j}\left(u_{0}, u_{1}, \ldots, u_{j-1}, \mu_{0}\right) .
$$


For example, $u_{1}$ satisfies the equation

$$
\mathcal{E}_{y}\left(y_{0}, \mu_{0}\right) y_{1}=-\mathcal{E}_{\mu}\left(u_{0}, \mu_{0}\right) .
$$

We note here that each $y_{j}$ can be obtained from its predecessors by solving a linear system with the same linear operator $\mathcal{E}_{y}\left(y_{0}, \mu_{0}\right)$. However, one cannot continue to use the same basis elements generated at fixed parameter $\mu^{*}$ to compute solutions when the parameter of interest is significantly away from it. From time to time, reduced basis elements have to be updated and the solution is sought in the new reduced basis space. Moreover, generating the right hand side of (1.2) could be quite complicated in certain problems. This choice has been extensively used in the literature, see for e.g [9], [10] for structural analysis problems and [14] for high Reynolds number steady state fluid flow calculations.

The Lagrange Subspace. In this case, the basis elements are solutions of the nonlinear problem under study at various parameter values $\mu_{j}$. The reduced subspace is given by

$$
X_{R}=\operatorname{span}\left\{y^{j} \mid y^{j}=y\left(\mu_{j}\right), j=1, \ldots, M\right\} .
$$

This kind of subspace was used to study structural problems in [1]. A possible advantage in this choice is that updating the basis elements can be done one basis vector at a time instead of generating the whole space.

The Hermite Subspace. This is a hybrid of the Lagrange and Taylor approach. The basis elements are solutions and their first derivatives at various parameter values $\mu_{j}$. The reduced subspace is given by

$$
X_{R}=\operatorname{span}\left\{y^{j}=y\left(\mu_{j}\right) \text { and }\left.\frac{\partial y}{\partial \mu}\right|_{\mu=\mu_{j}}, j=1, \ldots, \widetilde{M}\right\} .
$$

1.2. Reduced Basis Error. In this section we discuss the reduced basis error. Let $\mathrm{X}$ and $\mathrm{Y}$ be two Banach spaces and $\Lambda$ be a compact set in $\mathbb{R}^{p}$. Given a $C^{2}$ mapping

$$
\mathcal{E}:(y, \mu) \in X \times \Lambda \rightarrow \mathcal{E}(y, \mu) \in Y
$$

and we consider the equation

$$
\mathcal{E}(y, \mu)=0
$$

The family $\{(y(\mu), \mu): \mu \in \Lambda\}$ is said to be a branch of nonsingular solutions of equation (1.3), if,

$$
\begin{gathered}
\mu \rightarrow y(\mu) \text { is a continuous function from } \Lambda \text { into } X ; \\
\mathcal{E}(y, \mu)=0
\end{gathered}
$$

and $D_{y} \mathcal{E}(y, \mu)$ is an isomorphism from $X$ onto $Y$ for all $\mu \in \Lambda$. 
Let us consider the reduced order problem

$$
\mathcal{E}_{m}\left(y_{m}, \mu\right)=0, \quad y_{m} \in X_{m} \text { and } \mu \in \Lambda
$$

defined on the reduced basis space $X_{n}$. We assume that $\mathcal{E}_{m}: X_{m} \times \Lambda \rightarrow Y$ is $C^{2}$. For the ease of our discussions we assume that $X_{m} \subset X$ and $Y_{m} \subset Y$. The norms on $X_{m}$ and $Y_{m}$ are induced from $X$ and $Y$ norms, respectively. The problem is to find the solution $y_{m} \in X_{m}$ such that (1.4) is satisfied for a given $\mu \in \Lambda$.

We assume that $D_{y} \mathcal{E}_{m}\left(\tilde{y}_{m}, \mu\right)$ is an isomorphism from $X_{m}$ onto $Y_{m}$ where $\tilde{y}_{m}$ is a given element in $X_{m}$. We introduce the following notations;

$$
\begin{gathered}
\epsilon_{m}(\mu)=\left\|\mathcal{E}_{m}\left(\tilde{y}_{m}, \mu\right)\right\|_{Y}, \\
\gamma_{m}(\mu)=\left\|D_{y} \mathcal{E}_{m}\left(\tilde{y}_{m}, \mu\right)^{-1}\right\|_{\mathcal{L}(Y, X)}, \\
S_{m}(y ; \alpha)=\left\{v \in X_{m}:\|u-v\|_{X} \leq \alpha\right\}, \\
L_{m}(\mu ; \alpha)=\sup _{v \in S\left(\tilde{y}_{m}, \alpha\right)}\left\|D_{y} \mathcal{E}_{m}\left(\tilde{y}_{m}, \mu\right)-D_{y} \mathcal{E}_{m}(v, \mu)\right\|_{\mathcal{L}(X, Y)} .
\end{gathered}
$$

We next state a theorem regarding the error estimate which is derived from Theorem IV.3.1 in [6] for the approximation of branches of nonsingular solutions.

Theorem 1.1. Suppose $D_{y} \mathcal{E}_{m}\left(\tilde{y}_{m}, \mu\right)$ is an isomorphism of $X_{m}$ onto $Y_{m}$ and

$$
2 \gamma_{m}(\mu) L_{m}\left(\mu, 2 \gamma_{m}(\mu) \epsilon_{m}(\mu)\right)<1
$$

Then the problem (1.4) has a unique solution $\left(y_{m}(\mu), \mu\right)$ such that:

$$
y_{m}(\mu) \in S\left(\tilde{y}_{m} ; 2 \gamma_{m}(\mu) \epsilon_{m}(\mu)\right) .
$$

In addition, $y_{m}(\mu)$ is the only solution of (1.4) in the ball $S_{m}\left(\tilde{y}_{m} ; \alpha\right)$ for all $\alpha \geq 2 \gamma_{m}(\mu) \epsilon_{m}(\mu)$ that satisfy $\gamma_{m}(\mu) L_{m}(\mu ; \alpha)<1$ and we have the estimate:

$$
\left\|y_{m}(\mu)-v_{m}\right\|_{X} \leq\left[\gamma_{m}(\mu) /\left(1-\gamma_{m}(\mu) L_{m}(\mu ; \alpha)\right]\left\|\mathcal{E}_{m}\left(v_{m}, \mu\right)\right\|_{Y} \quad \text { for all } v_{m} \in S_{m}\left(\tilde{y}_{m}, \alpha\right) .\right.
$$

Moreover, we have the following corollary.

Corollary 1.2. Suppose there exists an element $\tilde{y}_{m} \in X_{m}$ such that $D_{y} \mathcal{E}\left(\tilde{y}_{m}, \mu\right)$ is an isomorphism of $X$ onto $Y$ and

$$
2 \gamma(\mu) L(\mu, 2 \gamma(\mu) \epsilon(\mu)<1
$$

where

$$
\epsilon(\mu)=\left\|\mathcal{E}\left(\tilde{y}_{m}, \mu\right)\right\|_{Y},
$$




$$
\begin{gathered}
\gamma(\mu)=\left\|D_{y} \mathcal{E}\left(\tilde{y}_{m}, \mu\right)^{-1}\right\|_{\mathcal{L}(Y, X)}, \\
S_{m}(y ; \alpha)=\left\{v \in X:\|u-v\|_{X} \leq \alpha\right\}, \\
L(\mu ; \alpha)=\sup _{v \in S\left(\tilde{y}_{m}, \alpha\right)}\left\|D_{y} \mathcal{E}\left(\tilde{y}_{m}, \mu\right)-D_{y} \mathcal{E}(v, \mu)\right\|_{\mathcal{L}(X, Y)} .
\end{gathered}
$$

Then the problem (1.1) has a solution $(y(\mu), \mu)$ such that:

$$
y(\mu) \in S\left(\tilde{y}_{m} ; 2 \gamma(\mu) \epsilon(\mu)\right) .
$$

In addition, $y(\mu)$ is the only solution of (1.1) in the ball $S\left(\tilde{y}_{m} ; \alpha\right)$ for all $\alpha \geq 2 \gamma(\mu) \epsilon(\mu)$ that satisfy $\gamma(\mu) L(\mu ; \alpha)<1$ and we have the estimate:

$$
\|y(\mu)-v\|_{X} \leq\left[\gamma(\mu) /(1-\gamma(\mu) L(\mu ; \alpha)]\|\mathcal{E}(v, \mu)\|_{Y} \quad \text { for all } v \in S\left(\tilde{y}_{m}, \alpha\right) .\right.
$$

We can apply Theorem 2.1 and Corollary 2.2 to obtain the following error estimate.

\section{REMARKS.}

(i) Suppose $y_{m}(\mu) \in X_{m}$ is a solution to (1.4) and assume $\tilde{y}_{m}=y_{m}(\mu)$ satisfies the condition in Corollary 2.2. Then we have a solution $y(\mu) \in S\left(\tilde{y}_{m} ; 2 \gamma(\mu) \epsilon(\mu)\right)$ to (1.3) and the estimate

$$
\left\|y(\mu)-y_{m}(\mu)\right\|_{X} \leq\left[\gamma(\mu) /(1-\gamma(\mu) L(\mu ; \alpha)]\left\|\mathcal{E}\left(y_{m}(\mu), \mu\right)\right\|_{Y}\right.
$$

(ii) Suppose there exits an element $\tilde{y}_{m} \in X_{m}$ such that the conditions in Theorem 2.1 and Corollary 2.2 are satisfied. Furthermore, we assume that $\alpha_{m}=2 \gamma_{m}(\mu) \epsilon_{m}(\mu)$ satisfies $\gamma(\mu) L\left(\mu ; \alpha_{m}\right)<1$. Then we have (1.7).

For related discussion on error analysis of reduced basis method, readers are referred to [4] and [15].

\section{THE REDUCED BASIS METHOD FOR NAVIER-STOKES FLOWS.}

Let us formulate the reduced basis method for viscous incompressible flows governed by the Navier-Stokes equations. The Navier-Stokes equations, when written in primitive variables, are

$$
\begin{gathered}
\mathbf{u}_{t}-\nu \Delta \mathbf{u}+\mathbf{u} \cdot \nabla \mathbf{u}+\nabla p=\mathbf{f} \quad \text { in } \Omega \times(0, T], \\
\nabla \cdot \mathbf{u}=0 \quad \text { in } \Omega \times(0, T], \\
\mathbf{u}=\mathbf{b} \quad \text { on } \Gamma \times[0, T],
\end{gathered}
$$

and

$$
\mathbf{u}(0, \mathbf{x})=\mathbf{u}_{0}(\mathbf{x}) \text { in } \Omega,
$$


where $\mathbf{u}(t, \mathbf{x})$ and $p(t, \mathbf{x})$ denote the velocity and pressure, respectively, $\mathbf{f}(t, \mathbf{x})$ the body force per unit mass, $\nu$ the kinematic viscosity and $\mathbf{u}_{0}$ the initial velocity. Furthermore $T$ is a positive constant, $\mathbf{b}$ is the boundary velocity and $\Omega$ is a bounded region in $\mathbb{R}^{2}$ whose boundary is $\Gamma$.

We choose variational formulation and finite element method to approximate (2.1) $-(2.4)$ but other methods can also be used with the reduced basis method. Casting (2.1)-(2.4) in appropriate variational form requires introduction of some notations.

2.1. Notations. We denote by $L^{2}(\Omega)$ the collection of square-integrable functions defined on $\Omega$ and we denote the associated norm by $\|\cdot\|_{0}$. Let

$$
\begin{gathered}
H^{1}(\Omega)=\left\{v \in L^{2}(\Omega): \frac{\partial v}{\partial x_{i}} \in L^{2}(\Omega) \text { for } i=1,2\right\}, \quad H_{0}^{1}(\Omega)=\left\{v \in H^{1}:\left.v\right|_{\partial \Omega}=0\right\}, \\
L_{0}^{2}(\Omega)=\left\{q \in L^{2}(\Omega): \int_{\Omega} q d \Omega=0\right\} \\
H^{m}(\Omega)=\left\{v \in L^{2}(\Omega): \frac{\partial^{|\alpha|} v}{\partial x_{1}^{\alpha_{1}} \partial x_{2}^{\alpha_{2}}} \in L^{2}(\Omega), \text { for all } \alpha=\left(\alpha_{1}, \alpha_{2}\right) \text { with }|\alpha| \leq m\right\}
\end{gathered}
$$

and we denote the norm on $H^{m}(\Omega)$ by $\|\cdot\|_{m}$. Vector-valued counterparts of these spaces are denoted by bold-face symbols, e.g., $\mathbf{H}^{1}=\left[H^{1}\right]^{2}$. Let $T>0$ and let $\mathbf{X}$ be a Banach space. We shall consider $L^{p}(0, T ; \mathbf{X}), 1 \leq p<\infty$, which is the space of functions from $[0, \mathrm{~T}]$ into $\mathrm{X}$ which are $L^{p}$ measurable for the measure dt. We equip with this the norm

$$
\left(\int_{0}^{T}\|\mathbf{u}(t)\|_{X}^{p} d t\right)^{\frac{1}{p}} .
$$

We also consider

$$
\mathbf{H}=\left\{\mathbf{v} \in \mathbf{L}^{2}(\Omega): \nabla \cdot \mathbf{v}=0 \text {, and }\left.\mathbf{v} \cdot \mathbf{n}\right|_{\Gamma}=0\right\}
$$

We define the following standard bilinear and trilinear forms associated with the NavierStokes problem

$$
\begin{gathered}
a(\mathbf{u}, \mathbf{v})=\int_{\Omega} \nabla \mathbf{u}: \nabla \mathbf{v} d \Omega \quad \text { for all } \mathbf{u}, \mathbf{v} \in \mathbf{H}^{1}(\Omega), \\
b(\mathbf{u}, q)=-\int_{\Omega} q \nabla \cdot \mathbf{u} d \Omega \quad \text { for all } \mathbf{u} \in \mathbf{H}^{1}(\Omega), \forall q \in L^{2}(\Omega)
\end{gathered}
$$

and

$$
c(\mathbf{u}, \mathbf{v}, \mathbf{w})=\frac{1}{2} \int_{\Omega}\{(\mathbf{u} \cdot \nabla) \mathbf{v} \cdot \mathbf{w}-(\mathbf{u} \cdot \nabla) \mathbf{w} \cdot \mathbf{v}\} d \Omega \quad \text { for all } \mathbf{u}, \mathbf{v}, \mathbf{w} \in \mathbf{H}^{1}(\Omega) .
$$


For given $\mathbf{b} \in \mathbf{H}^{\frac{1}{2}}(\Gamma)$ and the boundary condition

$$
\mathbf{u}=\mathbf{b} \text { on } \Gamma \text { with } \int_{\Gamma} \mathbf{b} \cdot \mathbf{n} d \Gamma=0
$$

we define

$$
\mathbf{V}_{\mathbf{b}}=\left\{\mathbf{u} \in \mathbf{H}^{1}(\Omega): \mathbf{u}=\mathbf{b} \text { on } \Gamma, \quad \mathbf{b} \in \mathbf{H}^{\frac{1}{2}}(\Gamma)\right\} .
$$

We now summarize some properties of these linear forms. We have the coercivity relations associated with $a(\cdot, \cdot)$ :

$$
a(\mathbf{u}, \mathbf{u})=\|\nabla \mathbf{u}\|_{0}^{2} \geq C_{0}\|\mathbf{u}\|_{1}^{2} \quad \text { for all } \mathbf{u} \in \mathbf{H}_{0}^{1}(\Omega)
$$

which is a direct consequence of Poincaré inequality. The forms $a(\cdot, \cdot), b(\cdot, \cdot)$ and $c(\cdot, \cdot, \cdot)$ are all continuous; in particular, we have

$$
|c(\mathbf{u}, \mathbf{v}, \mathbf{w})| \leq C_{1}\|\mathbf{u}\|_{1}\|\mathbf{v}\|_{1}\|\mathbf{w}\|_{1} .
$$

The bilinear form $b(\cdot, \cdot)$ satisfies the following inf-sup condition:

$$
\inf _{q \in L_{0}^{2}(\Omega)} \sup _{\mathbf{v} \in \mathbf{H}_{0}^{1}(\Omega)} \int_{\Omega} q \nabla \cdot \mathbf{v} d \Omega \geq C_{2} .
$$

2.2. Variational Formulation. We derive a variational formulation of the problem (2.1)-(2.4) by multiplying both sides of (2.1) and (2.2) by $\mathbf{v} \in \mathbf{H}_{0}^{1}(\Omega)$ and $q \in L^{2}(\Omega)$, respectively, and applying divergence theorem. We obtain Find $\mathbf{u} \in L^{2}\left(0, T ; \mathbf{V}_{\mathbf{b}}\right) \cap L^{\infty}(0, T ; \mathbf{H})$ and $p \in L^{2}\left(0, T ; L_{0}^{2}(\Omega)\right)$ such that

$$
\begin{gathered}
\left(\mathbf{u}_{t}, \mathbf{v}\right)+\frac{1}{R e} a(\mathbf{u}, \mathbf{v})+c(\mathbf{u}, \mathbf{u}, \mathbf{v})+b(\mathbf{v}, p)=(\mathbf{f}, \mathbf{v}) \quad \text { for all } \mathbf{v} \in \mathbf{H}_{0}^{1}(\Omega), \\
b(\mathbf{u}, q)=0 \quad \text { for all } q \in L_{0}^{2}(\Omega)
\end{gathered}
$$

and

$$
\mathbf{u}(0, \mathbf{x})=\mathbf{u}_{0}(\mathbf{x}) \quad \text { for } \mathbf{x} \in \Omega .
$$

Note here that the initial condition is required to satisfy the boundary conditions and the incompressibility condition. A typical finite element approximation of (2.5)-(2.6) is to seek solutions $\mathbf{u}^{h}(t, \cdot) \in \mathbf{V}_{\mathbf{b}}^{h} \subset \mathbf{V}_{\mathbf{b}}$ and $p^{h}(t, \cdot) \in S_{0}^{h} \subset L_{0}^{2}(\Omega)$

$$
\left(\mathbf{u}_{t}^{h}, \mathbf{v}^{h}\right)+\frac{1}{R e} a\left(\mathbf{u}^{h}, \mathbf{v}^{h}\right)+c\left(\mathbf{u}^{h}, \mathbf{u}^{h}, \mathbf{v}^{h}\right)+b\left(\mathbf{v}^{h}, p^{h}\right)=\left(\mathbf{f}, \mathbf{v}^{h}\right) \text { for all } \mathbf{v}^{h} \in \mathbf{V}_{0}^{h}
$$

and

$$
b\left(\mathbf{u}^{h}, q^{h}\right)=0 \text { for all } q^{h} \in S_{0}^{h},
$$

where $\mathbf{V}_{0}^{h} \subset \mathbf{H}_{0}^{1}(\Omega)$ and $S_{0}^{h} \subset L_{0}^{2}(\Omega)$ are approximating finite element subspaces, and we are setting $R e=1 / \nu$ which is the Reynolds number, i.e., the variables are appropriately nondimensionalized. We will denote the solution of the finite dimensional equations (2.7)$(2.8)$ at a fixed time $t_{i}$ by $\mathbf{u}^{h}\left(t_{i}, \cdot\right)$ and $p^{h}\left(t_{i}, \cdot\right)$. 


\subsection{The Reduced Basis Method and Reduced Order Model.}

2.3.1. Case I: Steady State. The Lagrange basis elements are generated by solving

$$
\frac{1}{R e} a\left(\mathbf{u}^{h}, \mathbf{v}^{h}\right)+c\left(\mathbf{u}^{h}, \mathbf{u}^{h}, \mathbf{v}^{h}\right)+b\left(\mathbf{v}^{h}, p^{h}\right)=\left(\mathbf{f}, \mathbf{v}^{h}\right) \quad \text { for all } \mathbf{v}^{h} \in \mathbf{V}_{0}^{h}
$$

and

$$
b\left(\mathbf{u}^{h}, q^{h}\right)=0 \quad \text { for all } q^{h} \in S_{0}^{h}
$$

for different values of parameter $\mu$, where $\mu=R e$. Thus, given a set of values for the Reynolds number $\left\{\mu_{i}: i=1,2,3, \ldots . M\right\}$, we solve (2.9)-(2.10) $M$ times to determine the set $\left\{\widehat{\mathbf{u}}_{m}: m=1, \ldots, M\right\}$, where $\widehat{\mathbf{u}}_{i}=\mathbf{u}^{h}\left(\mu_{i}\right)$. We then set

$$
\mathbf{V}^{M}=\operatorname{span}\left\{\widehat{\mathbf{u}}_{i}: i=1, \ldots, M\right\} \subset \mathbf{V}^{h} .
$$

We next briefly describe the Hermite approach in this setting. Let $\mathbf{u}_{i}=\mathbf{u}\left(\mu_{i}\right) \in \mathbf{V}^{h}$ and $\mathbf{u}_{i}^{\prime}=\frac{\partial \mathbf{u}^{h}}{\partial \mu}\left(\mu_{i}\right) \in \mathbf{V}_{0}^{h}$, then solve

$$
\frac{1}{\mu_{i}} a\left(\mathbf{u}_{i}^{\prime}, \mathbf{v}^{h}\right)+c\left(\mathbf{u}_{i}^{\prime}, \mathbf{u}_{0}, \mathbf{v}^{h}\right)+c\left(\mathbf{u}_{0}, \mathbf{u}_{i}^{\prime}, \mathbf{v}^{h}\right)+b\left(\mathbf{v}^{h}, p_{i}^{\prime}\right)=\frac{1}{\mu_{i}{ }^{2}} a\left(\mathbf{u}_{i}, \mathbf{v}^{h}\right) \quad \text { for all } \mathbf{v}^{h} \in \mathbf{V}_{0}^{h}
$$

and

$$
b\left(\mathbf{u}_{i}^{\prime}, q^{h}\right)=0 \quad \text { for all } q^{h} \in S_{0}^{h}
$$

to obtain $\mathbf{u}_{i}^{\prime}$. We then set

$$
V^{M}=\operatorname{span}\left\{\mathbf{u}_{i}, \mathbf{u}_{i}^{\prime}: i=1, \ldots, M\right\}
$$

Once we have a reduced basis functions we write the reduced order model in the form: seek $\mathbf{u}^{M} \in \mathbf{V}^{M} \subset \mathbf{V}^{h}$ such that

$$
\frac{1}{R e} a\left(\mathbf{u}^{M}, \mathbf{v}^{M}\right)+c\left(\mathbf{u}^{M}, \mathbf{u}^{M}, \mathbf{v}^{M}\right)=\left(\mathbf{f}, \mathbf{v}^{M}\right) \text { for all } \mathbf{v}^{M} \in \mathbf{V}_{0}^{M},
$$

where $\mathbf{V}_{0}^{m}=\mathbf{V}^{m} \cap \mathbf{V}_{0}^{h}$. Note that, by construction $\mathbf{u}^{M}$ automatically satisfies (2.10) and due to the global support of the reduced basis elements, the system (2.11) is equivalent to a dense lower order nonlinear system of equations as opposed to the system (2.9) $-(2.10)$ which is a sparse nonlinear system due to the local support of the basis. Our computational experiments and the computations reported for structural problems in the references mentioned earlier seem to indicate that an accurate approximation can be obtained for large range of parameter values using 5 to 10 basis elements. Therefore, although the resulting reduced order model is dense, they are small compared to the sparse but large system that result from the standard basis functions. 
2.3.2. Case II: Time Dependent State. A way to obtain reduced basis in this case is to solve (2.7)-(2.8) using implicit Euler method with appropriate step size at a given set of values for the time $\left\{t_{i}: i=1,2,3, \ldots . M\right\}$ to obtain the set $\left\{\widehat{\mathbf{u}}_{i}: i=1, \ldots, M\right\}$, where $\widehat{\mathbf{u}}_{i}=\mathbf{u}^{h}\left(t_{i}, \cdot\right)$. We then set $\mathbf{V}^{M}=\operatorname{span}\left\{\widehat{\mathbf{u}}_{i}: i=1, \ldots, M\right\}$. Once we have a reduced basis functions we write the reduced order model in the form: seek $\mathbf{u}^{M}(t, \cdot) \in \mathbf{V}^{M}=\operatorname{span}\left\{\mathbf{u}_{i}\right.$ : $i=1, \ldots ., M\} \subset \mathbf{V}^{h}$ such that

$$
\left(\frac{\partial}{\partial t} \mathbf{u}^{M}, \mathbf{v}^{M}\right)+\frac{1}{R e} a\left(\mathbf{u}^{M}, \mathbf{v}^{M}\right)+c\left(\mathbf{u}^{M}, \mathbf{u}^{M}, \mathbf{v}^{M}\right)=\left(\mathbf{f}, \mathbf{v}^{M}\right) \text { for all } \mathbf{v}^{M} \in \mathbf{V}_{0}^{M},
$$

where $\mathbf{V}_{0}^{M}=\mathbf{V}^{M} \cap \mathbf{V}_{0}^{h}$.

3. COMPUTATIONS OF THE REDUCED ORDER MODEL. In this section we will test the performance of reduced order model (2.11) in two well known test problems in fluid flows, namely cavity flow and backward-facing-step channel flow. Let us first consider the stationary case.

3.1. Stationary Driven Cavity Problem. The problem we are about to describe is a classical driven cavity flow. Various researchers have studied this problem computationally using variety of methods and formulations. We can think of this as fluid filled in a cavity bounded by rigid walls at $x=0, x=1, y=0$ and top wall is moving with unit speed. We consider of course a two dimensional situation and the domain is divided into rectangles and we further divide each rectangle into triangles and then choose quadratic polynomials defined on this triangles to approximate velocity fields and for the approximation of pressure we choose linear polynomial defined on the same triangles.

In all our computations reported in this article, we define Reynolds number as $R e=\frac{V \cdot L}{\nu}$. In the driven cavity problem, $V=$ top surface velocity, $\mathrm{L}=$ cavity dimension, $\nu=$ kinematic viscosity of the fluid. We assume throughout the simulations that $V=1, L=1$ and hence $R e=\frac{1}{\nu}$.

The computation using reduced basis method is done by first selecting basis elements and then defining test functions and trial functions such that they are linearly independent and the test functions satisfy homogeneous boundary conditions. We generate basis elements $\left\{\mathbf{u}_{i}\right\}_{i=1}^{M} \subset \mathbf{V}^{M}$ for reduced order model by computing the solutions at $M$ different Reynolds numbers to the full steady state Navier-Stokes equations

$$
\begin{gathered}
\frac{1}{R e} a\left(\mathbf{u}^{h}, \mathbf{v}^{h}\right)+c\left(\mathbf{u}^{h}, \mathbf{u}^{h}, \mathbf{v}^{h}\right)+b\left(\mathbf{v}^{h}, p^{h}\right)=\left(\mathbf{f}, \mathbf{v}^{h}\right) \text { for all } \mathbf{v}^{h} \in \mathbf{V}_{0}^{h} \\
b\left(\mathbf{u}^{h}, q^{h}\right)=0 \text { for all } q^{h} \in S_{0}^{h},
\end{gathered}
$$

and $\mathbf{u}=(1,0)$ on the top boundary and everywhere else on the boundary no slip boundary conditions is assumed.

Given the basis elements $\{\mathbf{u}\}_{i=1}^{M}$, reduced order solution $\mathbf{u}^{h}$ is formed by setting

$$
\mathbf{u}^{M}=\sum_{i=1}^{M} \alpha_{i} \phi_{i},
$$


where $\phi_{i}=\mathbf{u}_{i+1}-\mathbf{u}_{i}, i=1,2, . ., M-1$ and $\boldsymbol{\phi}_{M}=\mathbf{u}_{M}$. We further take $\alpha_{M}=1$ so that the boundary conditions are satisfied. The solution $\mathbf{u}^{M}$ is computed from

$$
\frac{1}{R e} a\left(\mathbf{u}^{M}, \mathbf{v}^{M}\right)+c\left(\mathbf{u}^{M}, \mathbf{u}^{M}, \mathbf{v}^{M}\right)=\left(\mathbf{f}, \mathbf{v}^{M}\right) \text { for all } \mathbf{v}^{M} \in \mathbf{V}_{0}^{M},
$$

where $\mathbf{V}_{0}^{M}=\operatorname{span}\left\{\phi_{i}: i=1, \ldots, M-1\right\}$ is the span of the test functions.

In our computations the basis elements for the reduced order model is obtained by computing the lid driven cavity flow at Reynolds numbers, 100, 300, 500, 700 and 900.

The computations are done with $29 \times 29$ nonuniform mesh. Comparison of reduced order model solution with the solution to the full model was done at Reynolds number 1200 and 1500. In Figures 1-2, computed solutions of driven cavity flow using reduced order model and the full model are plotted. We also studied the effects of the number of basis elements used in the reduced order model. The $l_{2}$-norm difference between the reduced and full solution is given in Tables I-II and a comparion of u-velocity along the vertical centerline of the cavity is given in Figures $4-5$.

Finally, we turn to a comparison study with Lagrange approach versus Hermite approach. The basis elements for the Lagrange approach were selected at Reynolds numbers $100,300,500$ and 700 , and that for the Hermite was selected at 300 and 700 . The comparison was carried out by computing the driven cavity flow at Reynolds number 1200 . For the Hermite approach the test function selection is as follows: $\phi_{1}=\mathbf{u}_{700}-\mathbf{u}_{300}, \boldsymbol{\phi}_{2}=\left.300 \mathbf{u}^{\prime}\right|_{300}$ and $\phi_{3}=\left.700 \mathbf{u}^{\prime}\right|_{700}$. The solution is then sought as

$$
\mathbf{u}=\mathbf{u}_{700}+\sum_{i=1}^{3} \alpha_{i} \boldsymbol{\phi}_{i} .
$$

The Figure 6 shows the u-velocity at the vertical centerline of the cavity using Hermite and Lagrange approaches. The $l_{2}$-norm difference between the full solution and the reduced basis solution using these two approaches are as follows: $\left|\mathbf{u}_{l}-\mathbf{u}_{f}\right|_{2}=0.0889$ and $\left|\mathbf{u}_{h}-\mathbf{u}_{f}\right|_{2}=$ 0.0766, where $\mathbf{u}_{l}$ is the solution obtained using Lagrange approach and $\mathbf{u}_{h}$ is that obtained using Hermite approach. According to our comparison with driven cavity problem, the performance of Hermite approach is better than that of Lagrange.

3.2. Unsteady Channel Problem. We demonstrate the feasibility of reduced basis method in unsteady problem by studying the channel flow past a backward-facing step. This problem has been extensively studied both experimentally and computationally. A schematic of the geometry is given in Figure 13. The height of the inflow boundary is 0.5 and that of the outflow boundary is 1 . The length of the narrower section of the channel is 1 and that of wider section of the channel is 7 (the total horizontal length is 8 ). We choose the viscosity constant $\nu=1 / 1000$. At the inflow we assume the flow is parabolic and we take $\mathbf{u}(y)=\mathbf{u}_{i}=8(y-0.5)(1-y)$. At outflow boundary, we again assume the flow is parabolic and $\mathbf{u}=\mathbf{u}_{0}=y(1-y)$. The prescribed body force $\mathbf{f}$ is chosen to be zero.

Triangular finite elements are chosen to discretize the domain. This choice is natural since we use nonuniform mesh with local refinements around the corner of the step and 
near the top of the no slip wall. The velocity and pressure are approximated by piecewise quadratic and piecewise linear polynomials, respectively.

We generate basis elements $\left\{\mathbf{u}_{i}\right\}_{i=1}^{M} \subset \mathbf{V}^{M}$ for reduced order model by computing the solutions at $\mathrm{M}$ different time instants to the full unsteady Navier-Stokes equations:

$$
\begin{gathered}
\left(\frac{\partial}{\partial t} \mathbf{u}^{h}, \mathbf{v}^{h}\right)+\frac{1}{R e} a\left(\mathbf{u}^{h}, \mathbf{v}^{h}\right)+c\left(\mathbf{u}^{h}, \mathbf{u}^{h}, \mathbf{v}^{h}\right)+b\left(\mathbf{v}^{h}, p^{h}\right)=\left(\mathbf{f}, \mathbf{v}^{h}\right) \text { for all } \mathbf{v}^{h} \in \mathbf{V}_{0}^{h} \\
b\left(\mathbf{u}^{h}, q^{h}\right)=0 \text { for all } q^{h} \in S_{0}^{h},
\end{gathered}
$$

and we assume fully developed flow at the inflow and outflow boundary, and everywhere else on the boundary no slip boundary conditions is assumed.

Given the basis elements $\left\{\mathbf{u}_{i}\right\}_{i=1}^{M}$, reduced order solution $\mathbf{u}^{M}$ is formed by setting

$$
\mathbf{u}^{M}(t)=\sum_{i=1}^{M} \alpha_{i}(t) \phi_{i},
$$

where $\phi_{i}=\mathbf{u}_{i+1}-\mathbf{u}_{i}, i=1,2, \ldots, M-1$ and $\phi_{M}=\mathbf{u}_{M}$. We further take $\alpha_{M}=1$ so that the boundary conditions are satisfied. The solution $\mathbf{u}^{M}$ is computed from

$$
\left(\frac{\partial}{\partial t} \mathbf{u}^{M}, \mathbf{v}^{M}\right)+\frac{1}{R e} a\left(\mathbf{u}^{M}, \mathbf{v}^{M}\right)+c\left(\mathbf{u}^{M}, \mathbf{u}^{M}, \mathbf{v}^{M}\right)=\left(\mathbf{f}, \mathbf{v}^{M}\right) \text { for all } \mathbf{v}^{M} \in \mathbf{V}_{0}^{M},
$$

where $\mathbf{V}_{0}^{M}=\operatorname{span}\left\{\boldsymbol{\phi}_{i}: i=1, \ldots, M-1\right\}$ is the span of the test functions.

For our computations the basis elements were generated by computing the flow from the full model at eleven time instances between 1 and 11. The time step used in the reduced order model was .001 and the computational domain was divided into triangles with refined grid near flow separation. Our computational experiment on backward facing step channel flow and on unsteady cavity flow (not reported here) indicates the clear and promising ability of the reduced order model in predicting the dynamics of fluid flows. The Figures 7-8 are the channel flow computations with full model and reduced order model at time $\mathrm{t}=10$, respectively.

4. CONTROL OF REDUCED ORDER MODEL. In order to develop the framework for the application of reduced basis method for the control of fluid flows, let us first formulate an optimal control problem.

$$
\text { Minimize } \mathcal{J}(\mathbf{u}, g)
$$

subject to

$$
\begin{gathered}
-\nu \Delta \mathbf{u}+\mathbf{u} \cdot \nabla \mathbf{u}+\nabla p=\mathbf{f} \text { in } \Omega, \\
\nabla \cdot \mathbf{u}=0 \quad \text { in } \Omega, \\
\left.\mathbf{u}\right|_{\Gamma_{1}}=\mathbf{b}
\end{gathered}
$$


and

$$
\left.\mathbf{u}\right|_{\Gamma_{2}}=g \boldsymbol{\tau}
$$

We discuss the boundary control problem and thus the body force is $\mathbf{f}$ is fixed. The function $g$ is the control input that influences the flow through the movement of part of the boundary $\Gamma_{2}$, the function $\mathbf{b}$ is a fixed boundary value on $\Gamma_{1}$ and $\tau$ is a unit tangential vector. We note here that this control mechanism is nondistructive in the sense that no mass is added into the system.

A variational form of (4.2)-(4.5) is defined as in the unsteady setting as

Find $\mathbf{u} \in \mathbf{V}_{\mathbf{b}}$ and $p \in L_{0}^{2}(\Omega)$ such that

$$
\frac{1}{R e} a(\mathbf{u}, \mathbf{v})+c(\mathbf{u}, \mathbf{u}, \mathbf{v})+b(\mathbf{v}, p)=(\mathbf{f}, \mathbf{v}) \quad \text { for all } \mathbf{v} \in \mathbf{H}_{0}^{1}(\Omega)
$$

and

$$
b(\mathbf{u}, q)=0 \quad \text { for all } q \in L_{0}^{2}(\Omega) .
$$

We will study two control problems that are cast in the framework of (4.1)-(4.5):

$\left(\mathcal{C}_{1}\right)$ The cavity control problem with the cost function

$$
\mathcal{J}(\mathbf{u}, g)=\int_{\Omega}\left|\mathbf{u}-\mathbf{u}_{d}\right|^{2} d \Omega .
$$

$\left(\mathcal{C}_{2}\right)$ The channel control problem with the cost function

$$
\mathcal{J}(\mathbf{u}, g)=\int_{\Omega}|\nabla \times \mathbf{u}|^{2} d \Omega
$$

Regarding the set of admissible controls $g$, we assume that the set $\mathcal{U}$ of admissible control $g$ is closed and bounded in $\mathbb{R}$.

Defining the set

$$
S=\left\{\mathbf{u} \in \mathbf{H}^{1}(\Omega): g \in \mathcal{U}, \mathbf{u} \text { satisfies }(4.2)-(4.5)\right\} .
$$

We have the following Theorem whose proof can be found in [3], [5] and [16].

Theorem 4.1. Suppose $\mathcal{U}$ is compact. Then $S$ is bounded in $\mathbf{H}^{1}(\Omega)$ and the control problems $\left(\mathcal{C}_{1}\right)$ and $\left(\mathcal{C}_{2}\right)$ have solutions.

Proof. An outline of the proof follows. First we define appropriate extensions $\mathbf{u}_{1}$ and $\mathbf{u}_{2}$ to the boundary values (4.4) and (4.5), respectively, and redefine (4.2)-(4.5) with a change of variable $\mathbf{u}=\mathbf{v}+g \mathbf{u}_{1}+\mathbf{u}_{2}$ such that the velocity $\mathbf{v}$ now satisfies homogeneous boundary values. Then estimating the terms in the variational form of (4.2)-(4.5) using the coercivity and continuity properties of the bilinear and trilinear forms and the antisymmetry property of the trilinear form. 
The second assertion follows from the observation that the cost functionals are weakly sequentially lower semicontinuous and bounded below by zero, the solution set $S$ is bounded in a Hilbert space $\mathbf{H}^{1}(\Omega)$, the set $\mathcal{U}$ is compact and $\mathbf{H}^{1}(\Omega)$ is compactly imbedded in $\mathbf{L}^{4}(\Omega)$. Then if we take a minimizing sequence $\left(\mathbf{u}_{n}, g_{n}\right) \in S \times \mathcal{U}$, there is a limit $\left(\mathbf{u}^{*}, g^{*}\right)$ to this sequence and the limit is in fact a minimum to the control problem.

To solve the control problems, we will use constrained minimization techniques based on the necessary condition of optimality. Let us first derive the necessary conditions of optimality for our control problems. To facilitate the forthcoming discussion we cast the control problems in the following abstract setting: For $(\mathbf{u}, g) \in \mathbf{H}_{0}^{1}(\Omega) \times \mathcal{U}$

$$
\begin{gathered}
\text { Minimize } \mathcal{J}(\mathbf{u}, g) \\
\text { subject to } \mathcal{G}(\mathbf{u}, p, g)=0 \text { and } \mathcal{H}(\mathbf{u})=0,
\end{gathered}
$$

where $\mathcal{G}(\mathbf{u}, p, g)=0$ now represents the Navier-Stokes contraint (4.6) and $\mathcal{H}(\mathbf{u})=0$ the divergence free condition (4.7). Then the Lagrangian can be written as

$$
\mathcal{L}(\mathbf{v}, p, g, \lambda, \sigma)=\mathcal{J}(\mathbf{u}, g)+\langle\boldsymbol{\lambda}, \mathcal{G}(\mathbf{u}, p, g)>+<\sigma, \mathcal{H}(\mathbf{u})>,
$$

where $\mathbf{u}=\mathbf{v}+g \mathbf{u}_{1}+\mathbf{u}_{2}, \lambda$ and $\sigma$ are Lagrange multipliers. The existence of Lagrange multipliers is guaranteed by the regular point condition, i.e. the linearized constraint is surjective. Before discussing the regular point condition further, let us define the variational form of the gradient of the constraints. Given $(\chi, q, h) \in \mathbf{H}_{0}^{1}(\Omega) \times L_{0}^{2}(\Omega) \times \mathbb{R}$,

$$
\begin{array}{r}
<\Psi, \mathcal{G}^{\prime}(\mathbf{u}, g)(\boldsymbol{\chi}, q, h)>+<r, \mathcal{H}^{\prime}(\mathbf{u})(\boldsymbol{\chi})>=a\left(\chi+h \mathbf{u}_{1}, \boldsymbol{\Psi}\right)+c\left(\boldsymbol{\chi}+h \mathbf{u}_{1}, \mathbf{u}, \Psi\right) \\
+c\left(\mathbf{u}, \boldsymbol{\chi}+h \mathbf{u}_{1}, \boldsymbol{\Psi}\right)+b(q, \boldsymbol{\Psi})+b\left(\boldsymbol{\chi}+h \mathbf{u}_{1}, r\right)
\end{array}
$$

for all $(\boldsymbol{\Psi}, r) \in \mathbf{H}_{0}^{1}(\Omega) \times L_{0}^{2}(\Omega)$. We then have the following equivalent solvability condition for the regular point condition:

Setting $\mathbf{\Phi}=\chi+h \mathbf{u}_{1}$ the solvability condition can be written as: given $\mathbf{s} \in \mathbf{H}^{-1}(\Omega)$ find $\Phi \in \mathbf{H}^{1}(\Omega)$ and $r \in L_{0}^{2}(\Omega)$ such that

$$
a(\boldsymbol{\Phi}, \boldsymbol{\Psi})+c(\boldsymbol{\Phi}, \mathbf{u}, \Psi)+c(\mathbf{u}, \boldsymbol{\Phi}, \Psi)+b(\bar{\Psi}, r)=<\mathbf{s}, \Psi>\text { for all } \boldsymbol{\Psi} \in \mathbf{H}_{0}^{1}(\Omega)
$$

and

$$
b(\Phi, q)=0 \quad \text { for all } q \in L_{0}^{2}(\Omega)
$$

The solvability of this system can be shown at least when data are small. Next as a result of the regular point condition [6], we have

THEOREM 4.2. Suppose the regular point condition is satisfied. Then we obtain the first order necessary condition for $(\mathbf{v}, p, g, \lambda, \sigma) \in \mathbf{H}_{0}^{1}(\Omega) \times L_{0}^{2}(\Omega) \times \mathbb{R} \times \mathbf{H}_{0}^{1}(\Omega) \times L_{0}^{2}(\Omega)$

$$
\frac{\partial \mathcal{L}}{\partial \mathbf{v}}(\chi)=a(\boldsymbol{\lambda}, \boldsymbol{\chi})+c(\boldsymbol{\chi}, \mathbf{u}, \boldsymbol{\lambda})+c(\mathbf{u}, \chi, \boldsymbol{\lambda})+b(\chi, \sigma)+<\mathcal{J}^{\prime}(\mathbf{u}), \chi>=0 \quad \text { for all } \chi \in \mathbf{H}_{0}^{1}(\Omega)
$$




$$
\frac{\partial \mathcal{L}}{\partial g}=a\left(\mathbf{u}_{1}, \boldsymbol{\lambda}\right)+c\left(\mathbf{u}, \mathbf{u}_{1}, \boldsymbol{\lambda}\right)+c\left(\mathbf{u}_{1}, \mathbf{u}, \boldsymbol{\lambda}\right)+b\left(\mathbf{u}_{1}, \sigma\right)+<\mathcal{J}^{\prime}(\mathbf{u}), \mathbf{u}_{1}>=0
$$

and

$$
\frac{\partial \mathcal{L}}{\partial p}(q)=b(\lambda, q)=0 \quad \text { for all } q \in L_{0}^{2}(\Omega)
$$

The system (4.6)-(4.10) characterizes the optimal control and optimal states and we call this optimality system.

4.1. Control of Driven Cavity Flow. In this section we formulate and numerically solve a control problem in driven cavity using reduced basis method. It is that of finding the bottom surface velocity $g$ such that the fluid velocity $\mathbf{u}$ is driven to a desired state $\mathbf{u}_{d}$. This control problem can be cast as a minimization problem with the cost function

$$
\mathcal{J}(\mathbf{u})=\int_{\Omega}\left|\mathbf{u}-\mathbf{u}_{d}\right|^{2} d \Omega
$$

and subject to the constraint that the fluid obeys the equation of motion, where $\mathbf{u}_{d}$ is the desired velocity field.

The geometry of the problem and the finite element approximations have already been discussed in $\S 3.1$. Replacing the cost function in $\left(\mathcal{C}_{1}\right)$ in the abstract problem, the control problem for the driven cavity is written as:

$$
\text { Minimize } \mathcal{J}(\mathbf{u})=\int_{\Omega}\left|\mathbf{u}-\mathbf{u}_{d}\right|^{2} d \Omega
$$

subject to

$$
\frac{1}{R e} a(\mathbf{u}, \mathbf{v})+c(\mathbf{u}, \mathbf{u}, \mathbf{v})+b(\mathbf{v}, p)=(\mathbf{f}, \mathbf{v}) \quad \text { for all } \mathbf{v} \in \mathbf{H}_{0}^{1}(\Omega)
$$

and

$$
\begin{gathered}
b(\mathbf{u}, q)=0 \quad \text { for all } q \in L_{0}^{2}(\Omega), \\
\left.\mathbf{u}\right|_{\Gamma_{\text {top }}}=\left(U^{\text {top }}, 0\right),\left.\quad \mathbf{u}\right|_{\Gamma_{\text {bot }}}=(g, 0), \quad \text { and }\left.\quad \mathbf{u}\right|_{\Gamma_{\text {side }}}=(0,0),
\end{gathered}
$$

where $U^{\text {top }}, g$ are top and bottom surface velocities respectively. We wish to find the control input $g$ such that the flow matches as close as possible to a desired flow $\mathbf{u}_{d}$. The top velocity is fixed throughout the problem. Figure 9 gives the physical domain and the boundary conditions.

Using the reduced basis method, we now consider the reduced order control problem for driven cavity in the following form:

$$
\text { Minimize } \mathcal{J}\left(\mathbf{u}^{M}, g\right)
$$


subject to

$$
\frac{1}{R e} a\left(\mathbf{u}^{M}, \mathbf{v}^{M}\right)+c\left(\mathbf{u}^{M}, \mathbf{u}^{M}, \mathbf{v}^{M}\right)=\left(\mathbf{f}, \mathbf{v}^{M}\right) \text { for all } \mathbf{v}^{M} \in \mathbf{V}_{0}^{M} .
$$

Basis elements are computed with the boundary conditions described in Table III. The test functions $\left\{\boldsymbol{\phi}_{1}, \boldsymbol{\phi}_{2}\right\}$ are chosen so that they have zero boundary conditions. The trial function $\phi_{3}=\mathbf{u}_{4}$ corresponds to the control force such that $\left.\phi_{3}\right|_{\Gamma_{\text {bot }}} \neq 0$ and satisfies zero boundary conditions everywhere else. Then we seek the solution as

$$
\mathbf{u}^{M}=\mathbf{u}_{1}+g \mathbf{u}_{4}+\sum_{i=1}^{2} \alpha_{i} \phi_{i}
$$

where $g$ is the control (tangential velocity at the boundary) and, $\phi_{1}=\mathbf{u}_{2}-\mathbf{u}_{1}-\mathbf{u}_{4}$ and $\phi_{2}=\mathbf{u}_{3}-\mathbf{u}_{1}+\mathbf{u}_{4}$. We take $\mathbf{V}_{0}^{M}=\operatorname{span}\left\{\boldsymbol{\phi}_{1}, \phi_{2}\right\}$.

The computation of optimal control is carried out in two steps: First the necessary conditions of optimality system (4.6)-(4.10) is derived for this problem. Then this system is solved by applying Newton's method. The computations for this problem were done with $29 \times 29$ nonuniform mesh and the Reynolds number was $500(\nu=1 / 500)$. The top wall velocity is taken to be $U^{\text {top }}=1$ and the desired velocity $\mathbf{u}_{d}$ is computed with the bottom wall moving at one half of the top wall velocity. We get control $g^{\text {opt }}=0.4806$ in 4 Newton iterations and the corresponding boundary velocity therefore is 0.4806 . The resulting flow field is given in Figure 11. We also carried out computations to find the flow field corresponding to the optimal control input computed from the reduced order model which is given in Figure 12. They all are in good agreement with the desired flow field given in Figure 10.

4.2. Control of Channel Flows. In this section, we consider the problem of control of channel flows. We will consider two different geometrical configurations, namely the forward facing step and the backward facing step, a schematic of these geometries are given in Figure 13 and Figure 17. The aim is to shape the flow to a desired configuration by means of controlled movement of boundary along some part of the boundary. In this work we consider the minimization of vorticity in the flow. Thus we consider the following cost functional:

$$
\mathcal{J}(\mathbf{u}, g)=\int_{\Omega}|\nabla \times \mathbf{u}|^{2} d \Omega
$$

where the vorticity $\boldsymbol{\omega}=\nabla \times \mathbf{u}$. The control problem for the channel is posed in the following form:

$$
\text { Minimize } \mathcal{J}(\mathbf{u})=\int_{\Omega}|\nabla \times \mathbf{u}|^{2} d \Omega
$$

subject to

$$
\frac{1}{R e} a(\mathbf{u}, \mathbf{v})+c(\mathbf{u}, \mathbf{u}, \mathbf{v})+b(\mathbf{v}, p)=(\mathbf{f}, \mathbf{v}) \quad \text { for all } \mathbf{v} \in \mathbf{H}_{0}^{1}(\Omega)
$$




$$
\begin{gathered}
b(\mathbf{u}, q)=0 \quad \text { for all } q \in L_{0}^{2}(\Omega), \\
\left.\mathbf{u}\right|_{\Gamma_{1}}=\mathbf{b} \quad \text { and }\left.\quad \mathbf{u}\right|_{\Gamma_{2}}=g \boldsymbol{\tau},
\end{gathered}
$$

where $\Gamma_{2}$ is part of the boundary where boundary surface is moving (control input) and $\Gamma_{2}$ is rest of the boundary. Then $\mathbf{b} \in \mathbf{H}^{\frac{1}{2}}(\Gamma)$ corresponds to the inflow, outflow boundary conditions and zero boundary conditions at the walls. Also, $g$ is the magnitude of the boundary surface velocity. In the following we will consider two channel geometries and in each of them our choice of control portion $\Gamma_{2}$ is not the only one possible. But it is motivated by the fact that if one wants maximum influence in the flow, then the control has to be applied in that vicinity.

4.2.1. Case I: Backward-Facing-Step Channel Flow. First we consider a control problem in a backward facing step channel flow. We assume that the inflow and outflow are parabolic as elaborated in $\$ 3.2$. Figure 15 qualitatively demonstrate the situation for high Reynolds number. As mentioned previously, the aim is to shape the flow to a desired configuration by controlled boundary movement. A desirable flow of course depends on the situation in which the flow occurs. Here our objective is to remove the recirculation that occurs in the corner region. Thus the control portion $\Gamma_{2}$ is taken to be the line segment between $y=0$ and $y=0.5$ at $x=1$ where we note that at $x=1$ is where the channel changes its cross section area. Also, we take $\boldsymbol{\tau}=(1,0)$, that is the movement of the wall is vertical and thus $g \in \mathbb{R}$ completely determines the control input.

Basis elements are computed with the boundary conditions tabulated in Table IV. The test functions $\left\{\boldsymbol{\phi}_{1}, \ldots, \phi_{4}\right\}$ are chosen so that they have zero boundary conditions. The trial function $\phi_{5}$ corresponds to the control force such that $\left.\phi_{5}\right|_{\Gamma_{1}}=0$ and $\left.\phi_{5}\right|_{\Gamma_{2}} \neq 0$. Then we set

$$
\mathbf{u}=\mathbf{u}_{1}+g \phi_{5}+\sum_{i=1}^{4} \alpha_{i} \phi_{i},
$$

where $\phi_{1}=\mathbf{u}_{3}-2 \mathbf{u}_{2}+\mathbf{u}_{1}, \phi_{2}=\mathbf{u}_{4}-3 \mathbf{u}_{2}+2 \mathbf{u}_{1}, \phi_{3}=\mathbf{u}_{5}-4 \mathbf{u}_{2}+3 \mathbf{u}_{1}, \phi_{4}=\mathbf{u}_{6}-5 \mathbf{u}_{2}+4 \mathbf{u}_{1}$ and $\phi_{5}=\mathbf{u}_{6}-\mathbf{u}_{1}$. Then, for the vorticity cost functional $\left(\mathcal{C}_{2}\right)$, with the Reynolds number $200(\nu=1 / 200)$, we obtain the optimal control $g^{\text {opt }}=0.2601$ in 5 Newton iterations and the corresponding optimal boundary velocity therefore is -0.13005 . The resulting flow is shown in Figure 14. We also simulated the flow corresponding to the optimal control computed from the reduced order model and the result is shown in Figure 16. The results show significant reduction in the corner circulation.

4.2.2. Case II: Forward-Facing-Step Channel Flow. The second case we investigate is the control of forward facing step channel flow. We assume that the inflow and outflow are parabolic with $u(y)=u_{i}=y(1-y / 3) / 3$ and $u(y)=u_{o}=3(3-y)(y-1) / 8$, respectively. Figure 18 qualitatively demonstrate the situation for high Reynolds number. Our objective in this case is to remove the recirculation that occurs on the top of the step. The boundary conditions with which the basis elements computed are tabulated in Table 
$V$. The test functions $\left\{\boldsymbol{\phi}_{1}, \ldots, \phi_{4}\right\}$ are chosen so that they have zero boundary conditions. The trial function $\phi_{5}$ corresponds to the control force such that $\left.\phi_{5}\right|_{\Gamma_{1}}=0$ and $\left.\phi_{5}\right|_{\Gamma_{2}} \neq 0$. Then we set

$$
\mathbf{u}=\mathbf{u}_{1}+g \phi_{5}+\sum_{i=1}^{4} \alpha_{i} \phi_{i},
$$

where $\phi_{1}=\mathbf{u}_{6}-3 \mathbf{u}_{2}+2 \mathbf{u}_{1}, \phi_{2}=\mathbf{u}_{6}-2 \mathbf{u}_{3}+\mathbf{u}_{1}, \phi_{3}=\mathbf{u}_{6}-1.5 \mathbf{u}_{4}+.5 \mathbf{u}_{1}, \phi_{4}=\mathbf{u}_{6}-1.2 \mathbf{u}_{5}+.2 \mathbf{u}_{1}$ and $\phi_{5}=\mathbf{u}_{6}-\mathbf{u}_{1}$.

We take the control region to be the line segment between $x=1$ and $x=5$ at $y=3$ here we note that at $y=3$ is where the channel changes its cross section area. Also, we take $\boldsymbol{\tau}=(1,0)$, that is the movement of the wall is horizontal and like in the previous case $g \in \mathbb{R}$ completely determines the control input.

Then, for the vorticity cost $\left(\mathcal{C}_{2}\right)$, with the Reynolds number $1000(\nu=1 / 1000)$, we obtain the optimal control $g^{\text {opt }}=0.3041$ in 17 Newton iterations and the corresponding optimal boundary velocity therefore is 0.09120 . The resulting flow is shown in Figure 19. We also simulated the flow corresponding to the optimal control computed from the reduced order model and the result is shown in Figure 20. The results show significant reduction in the corner circulation.

5. CONCLUSION. Reduced basis method for simulation and control of fluid flow problems is presented. Numerical results are given for driven cavity and channel flow control problems in steady viscous incompressible flows. Our computational clearly demonstrate the performance of reduced order model for fluid flow control problems in several geometrical configurations. Feedback control for fluid dynamics based on reduced basis method are currently underway and will be reported in a forthcoming article. 


\section{REFERENCES}

[1] B.O. Almroth, P. Stern and F.A. Brogan, AiAA Journal, 16, 525 (1978).

[2] F. Brezzi, J. Rappaz, P.A. Raviart, Numer. Math. 36, 1 (1980).

[3] M. Desai and K. ITo, SIAM J. Control Es Optimization 32, 1428 (1994).

[4] J. Fink and W. Rheinboldt, $Z A M M, \mathbf{6 3}, 21$ (1983).

[5] H.O. Fattorini and S. S. Sritharan, Proceeding of the Royal Socity of London, Series A, 43981 (1992).

[6] V. Girault and P. Raviart, Finite Element Methods for Navier-Stokes Equations (Springer-Verlag, New York, 1986).

[7] K. Ito and S.S. Ravindran, Proceedings of the 35th $C D C$, IEEE, Kobe, Japan, 1996.

[8] H. Mauer and J. Zowe, Math. Programming, 16, 98 (1979).

[9] D. NAGY, Computers \& Structures, 10, 683 (1979).

[10] A.K. Noor, Computers \& Structures, 13, 31 (1981).

[11] A.K. Noor, C.M. Anderson and J.M. Peters, AIAA Journal, 19, 393 (1981).

[12] A.K. Noor and J.M. Peters, AIA A Journal, 18, 455 (1980).

[13] A.K. Noor and J.M. Peters, Comp. Meth. Appl. Mech. Eng., 28, 217 (1981).

[14] J.S.Peterson, SIAM J.Sci. Stat. Comput., 10777 (1989).

[15] T. Porsching, Math. Comp., 45487 (1985).

[16] S. S. SRItharan, System and Control Letters, 16299 (1991). 


\begin{tabular}{|l||c|c|c|c|}
\hline \hline Basis elements & 2 & 3 & 4 & 5 \\
\hline$\left|\mathbf{u}_{r}-\mathbf{u}_{f}\right|_{2}$ & .3989 & .06913 & .0600 & .04322 \\
\hline \hline
\end{tabular}

TABLE I. $l_{2}$ difference of solutions at $\operatorname{Re}=1200$

\begin{tabular}{|l||c|c|c|c|}
\hline \hline Basis elements & 2 & 3 & 4 & 5 \\
\hline$\left|\mathbf{u}_{r}-\mathbf{u}_{f}\right|_{2}$ & .5504 & .0729 & .0698 & .0545 \\
\hline \hline
\end{tabular}

TABLE II. $l_{2}$ difference between solutions at $\operatorname{Re}=1500$

\begin{tabular}{|l||c|c|c|c|}
\hline \hline Basis elements & $\mathbf{u}_{1}$ & $\mathbf{u}_{2}$ & $\mathbf{u}_{3}$ & $\mathbf{u}_{4}$ \\
\hline Top wall velocity & 1 & 1 & 1 & 0 \\
\hline Bottom wall velocity & 0 & 1 & -1 & 1 \\
\hline \hline
\end{tabular}

TABLE III. Wall velocities for basis vector generation

\begin{tabular}{|l||c|c|c|c|c|c|}
\hline \hline Basis elements & $\mathbf{u}_{1}$ & $\mathbf{u}_{2}$ & $\mathbf{u}_{3}$ & $\mathbf{u}_{4}$ & $\mathbf{u}_{5}$ & $\mathbf{u}_{6}$ \\
\hline Wall velocity & 0 & -0.1 & -0.2 & -0.3 & -0.4 & -0.5 \\
\hline \hline
\end{tabular}

TABLE IV. Wall velocities for basis vector generation

\begin{tabular}{|l||c|c|c|c|c|c|}
\hline \hline Basis elements & $\mathbf{u}_{1}$ & $\mathbf{u}_{2}$ & $\mathbf{u}_{3}$ & $\mathbf{u}_{4}$ & $\mathbf{u}_{5}$ & $\mathbf{u}_{6}$ \\
\hline Wall velocity & 0 & 0.1 & 0.15 & 0.2 & 0.25 & 0.3 \\
\hline \hline
\end{tabular}

TABLE V. Wall velocities for basis vector generation 


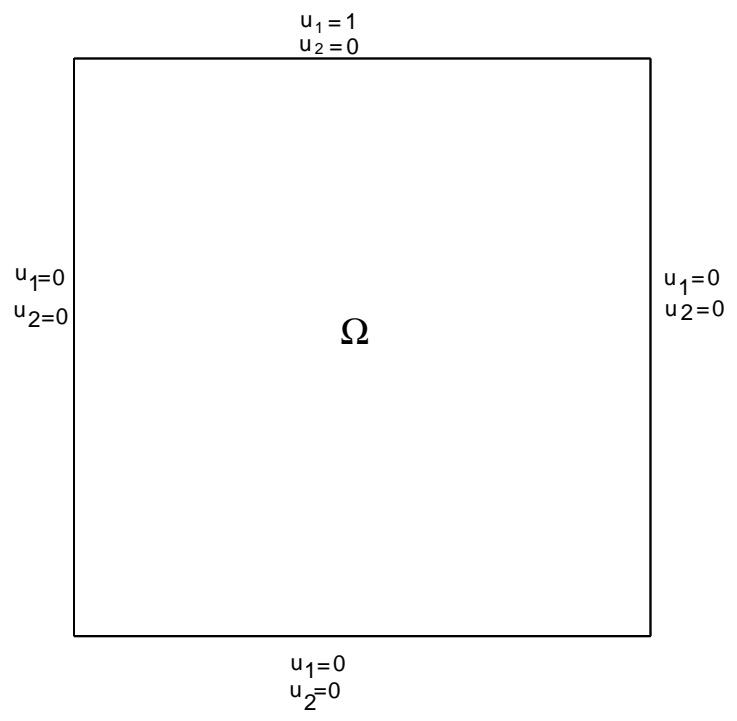

FIG. 1. Schematic of driven cavity

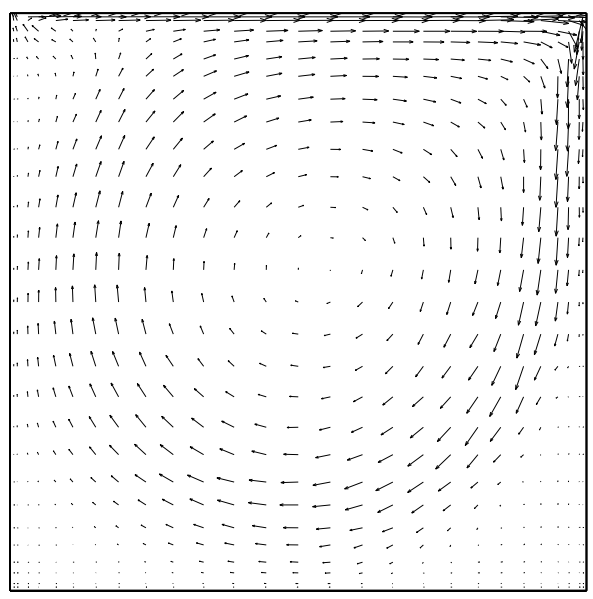

FIG. 2. Reduced solution with 4 basis: Re $=1200$ 


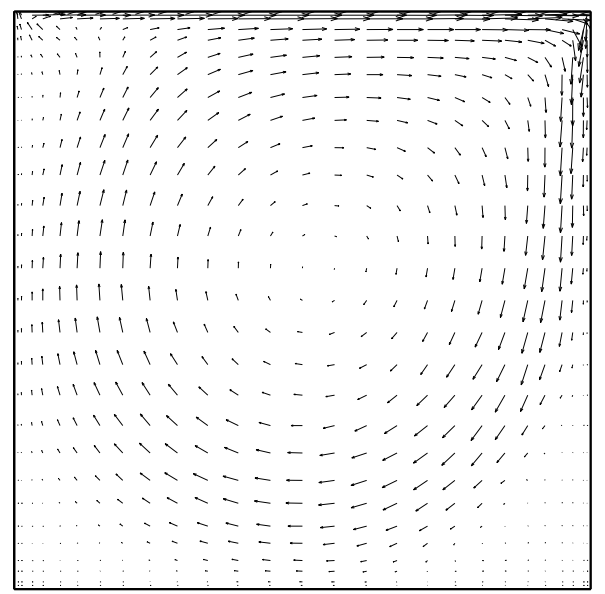

FIG. 3. Solution to full system: $\operatorname{Re}=1200$

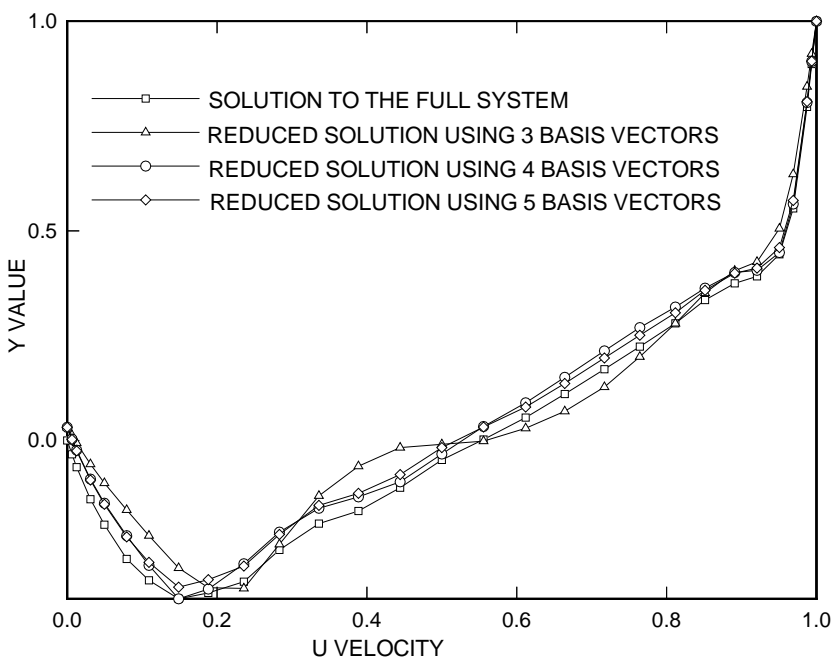

FIG. 4. Comparison of reduced basis solution to full solution at $R e=1200$ 


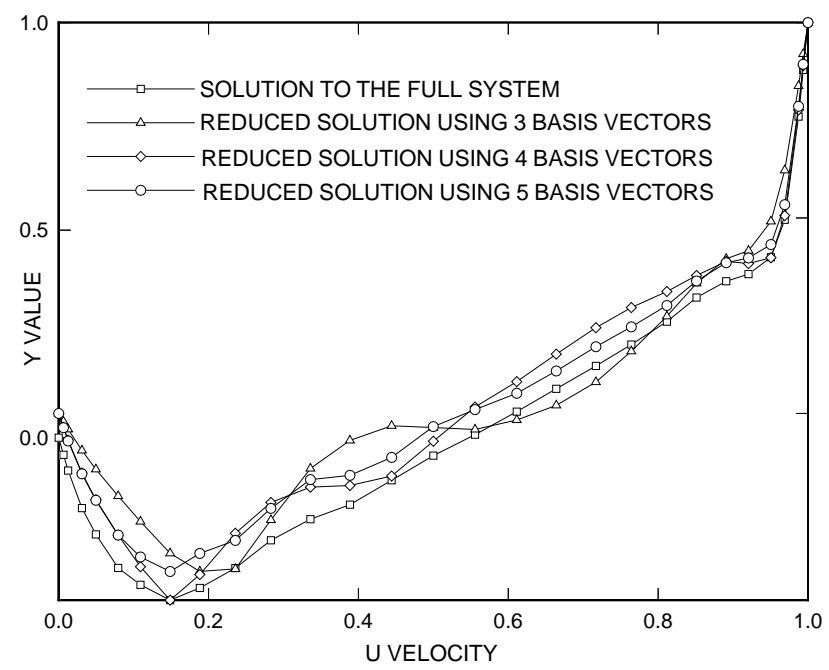

FIG. 5. Comparison of reduced basis solution to full solution at $\operatorname{Re}=1500$

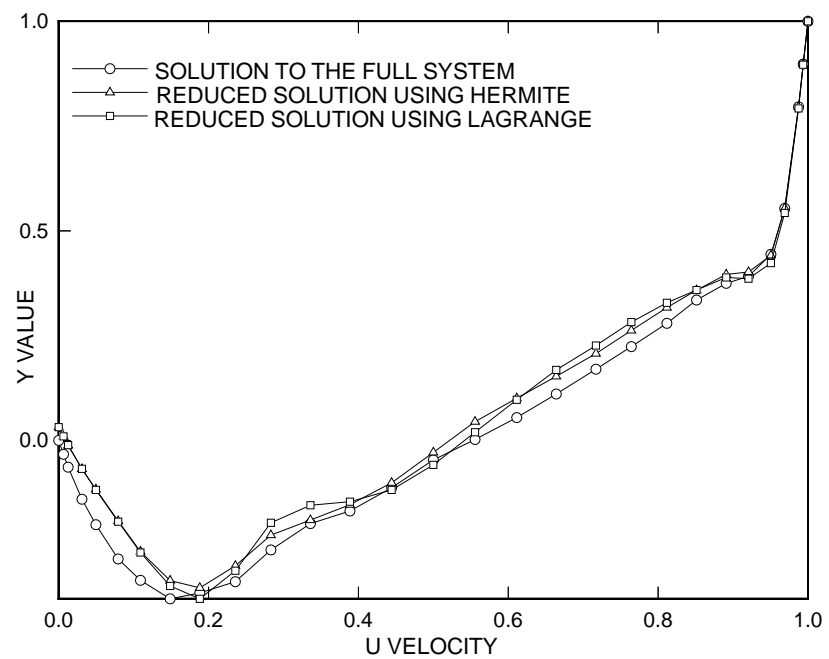

FIG. 6. Comparison of Hemite solution to Lagrange solution with 4 basis elements at Re=1200 


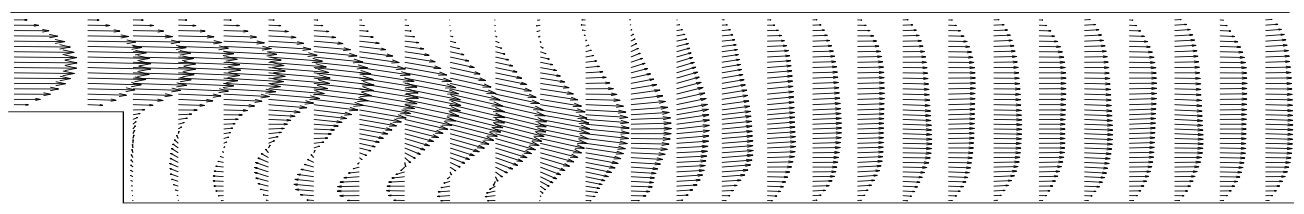

FIG. 7. Full solution when $\mathrm{t}=10$ and $\mathrm{Re}=1000$

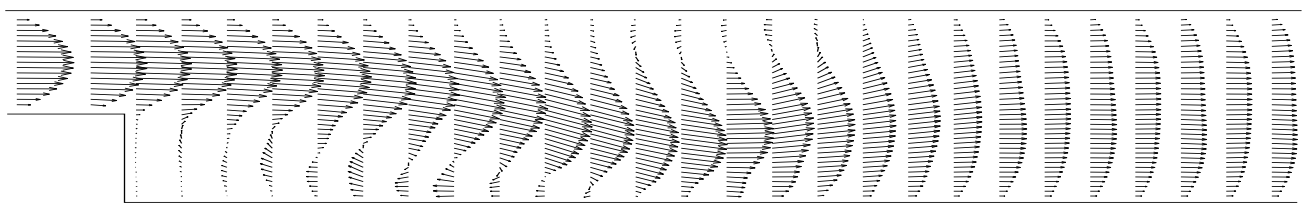

FIG. 8. Reduced basis solution when $t=10$ and $R e=1000$ 


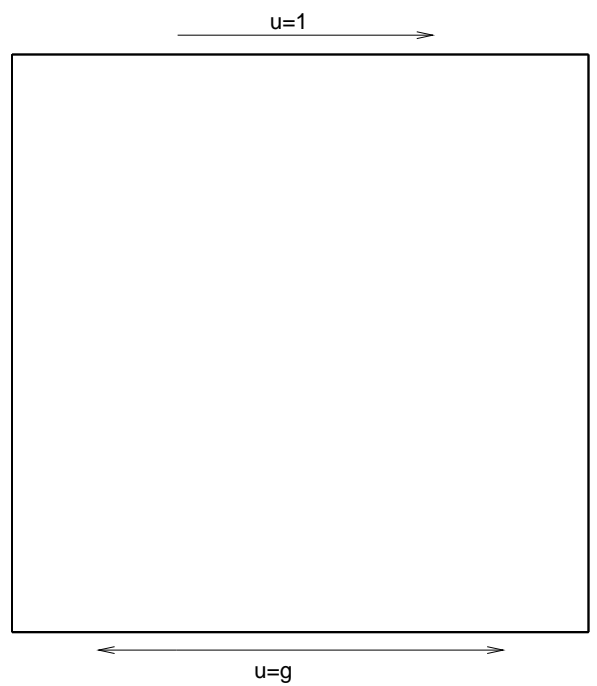

FIG. 9. Schematic of controlled driven cavity

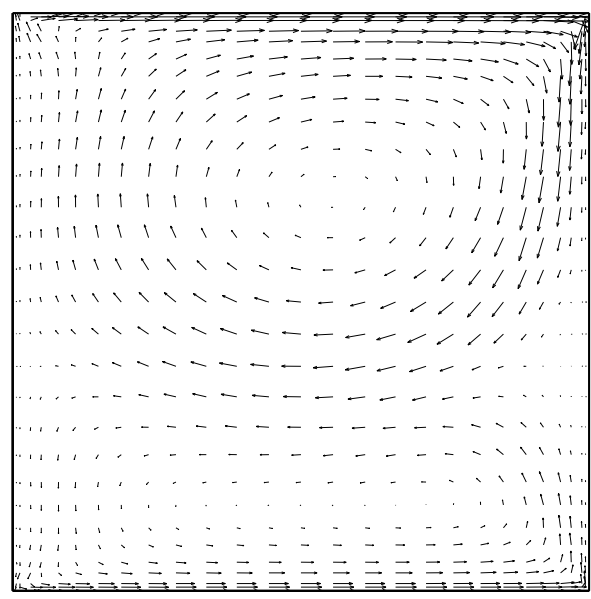

FIG. 10. Desired velocity field at $\mathrm{Re}=500$ 


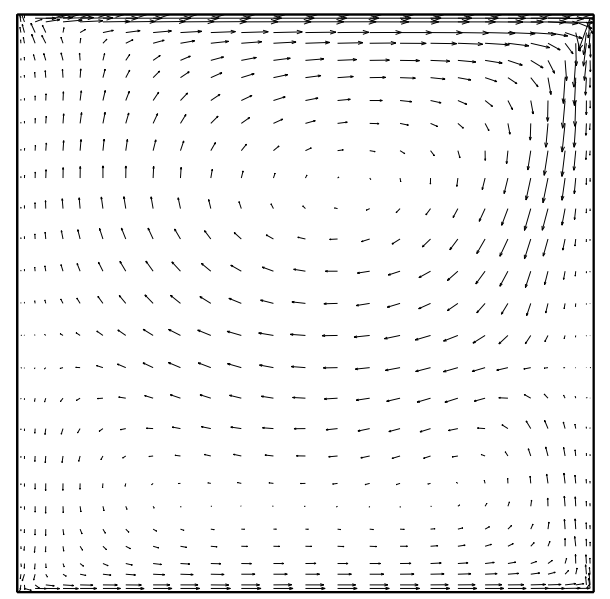

FIG. 11. Controlled velocity field at $R e=500$

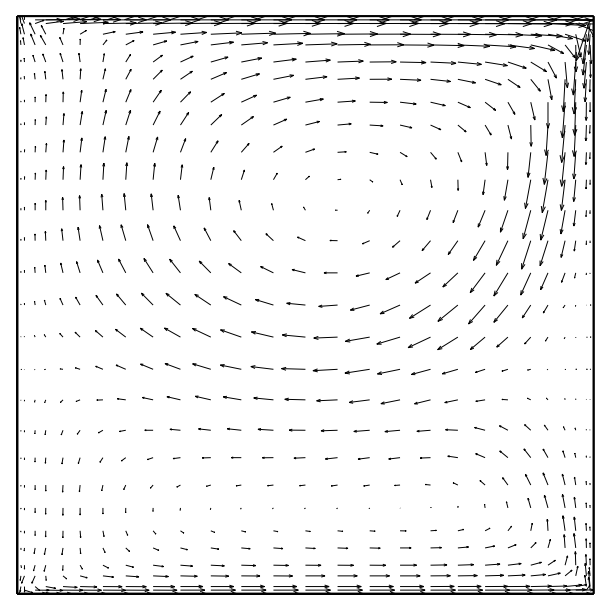

FIG. 12. Cavity flow with optimal control input at Re=500 


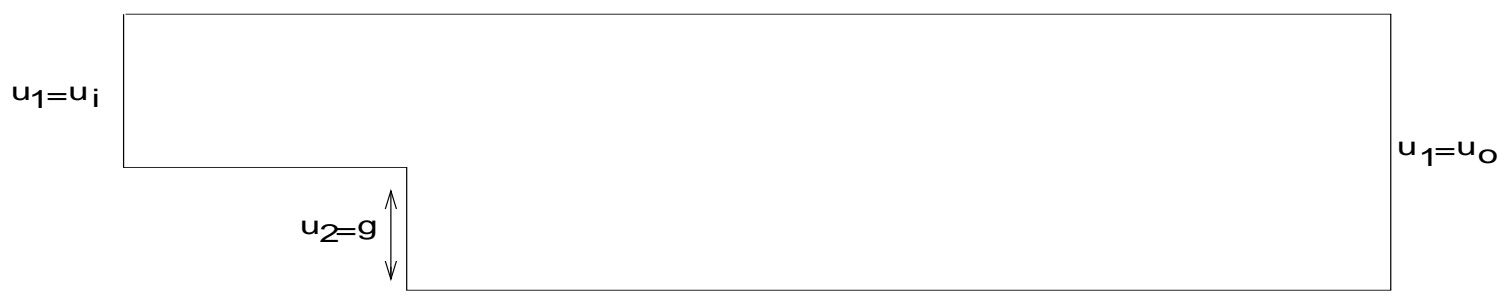

FIG. 13. Schematic of controlled backward-facing-step channel

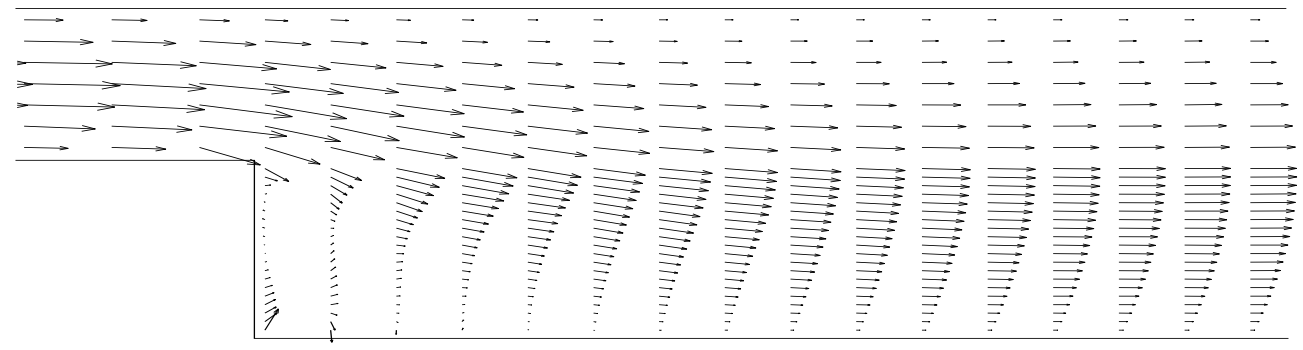

FIG. 14. Controlled velocity field at $R e=200$ 


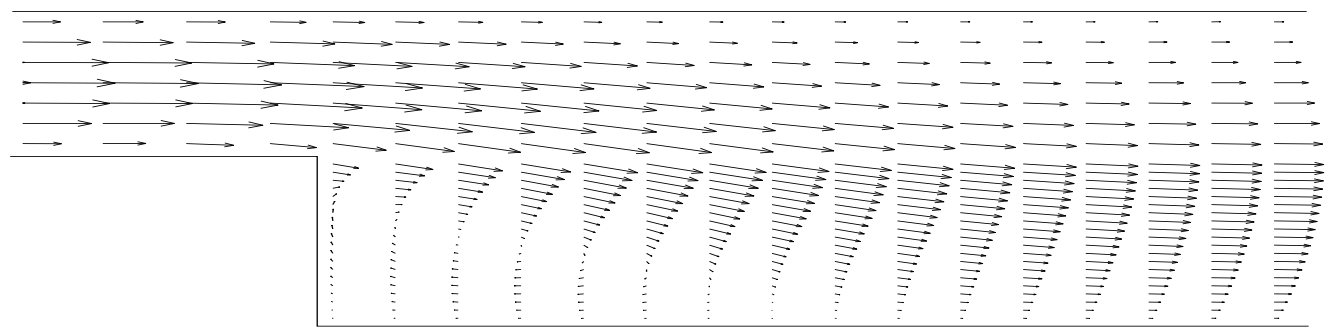

FIG. 15. Uncontrolled velocity field at $\mathrm{Re}=200$

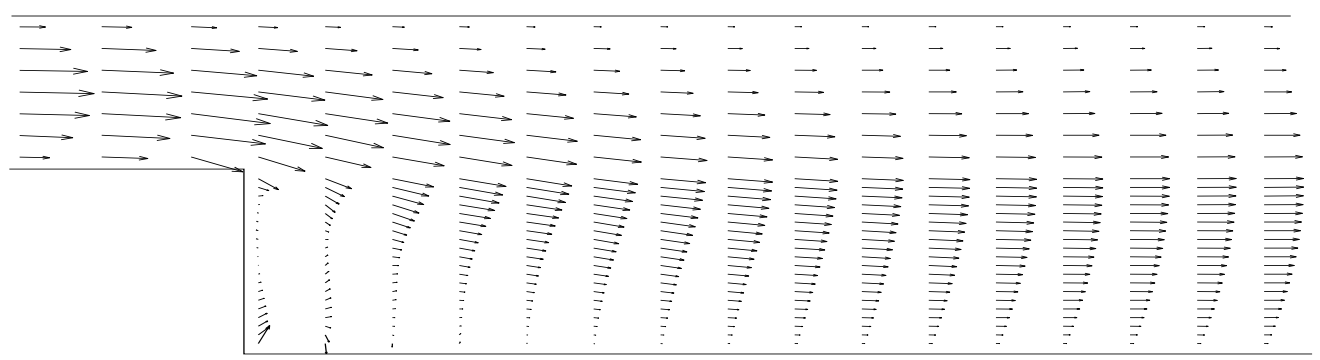

FIG. 16. Channel flow with optimal control input at $R e=200$ 


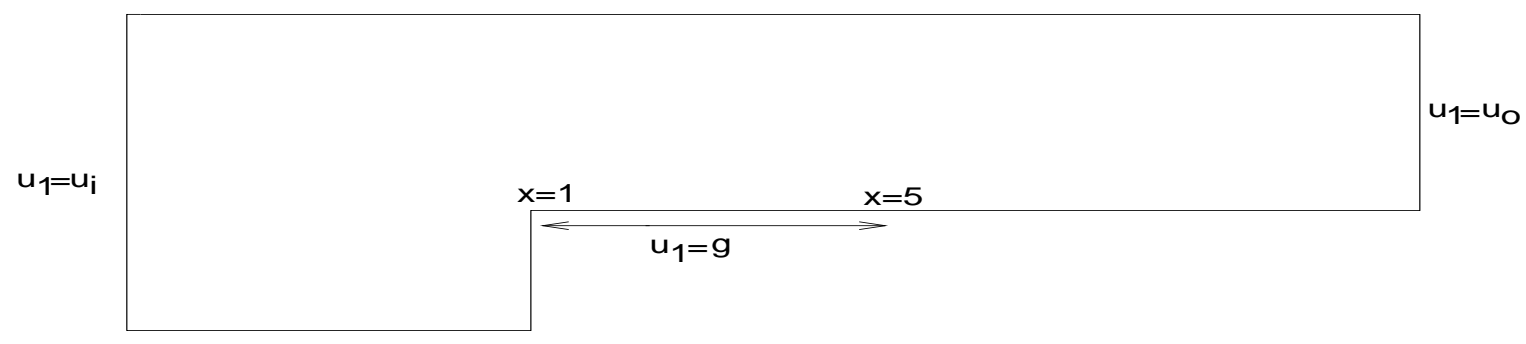

FIG. 17. Schematic of controlled forward-facing-step channel

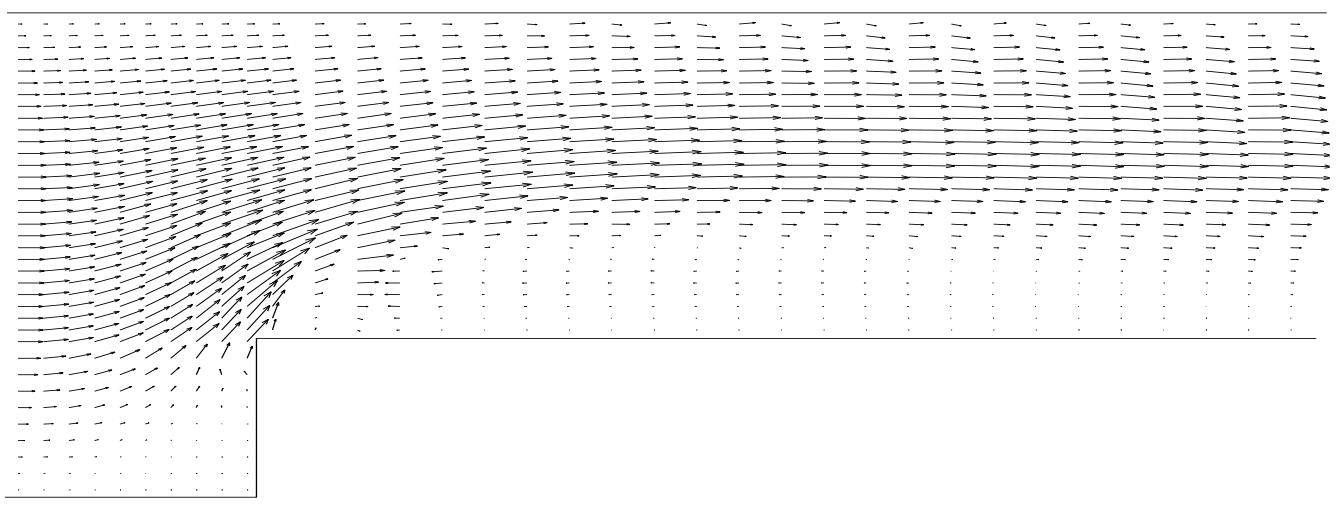

FIG. 18. Uncontrolled velocity field at $R e=1000$ 


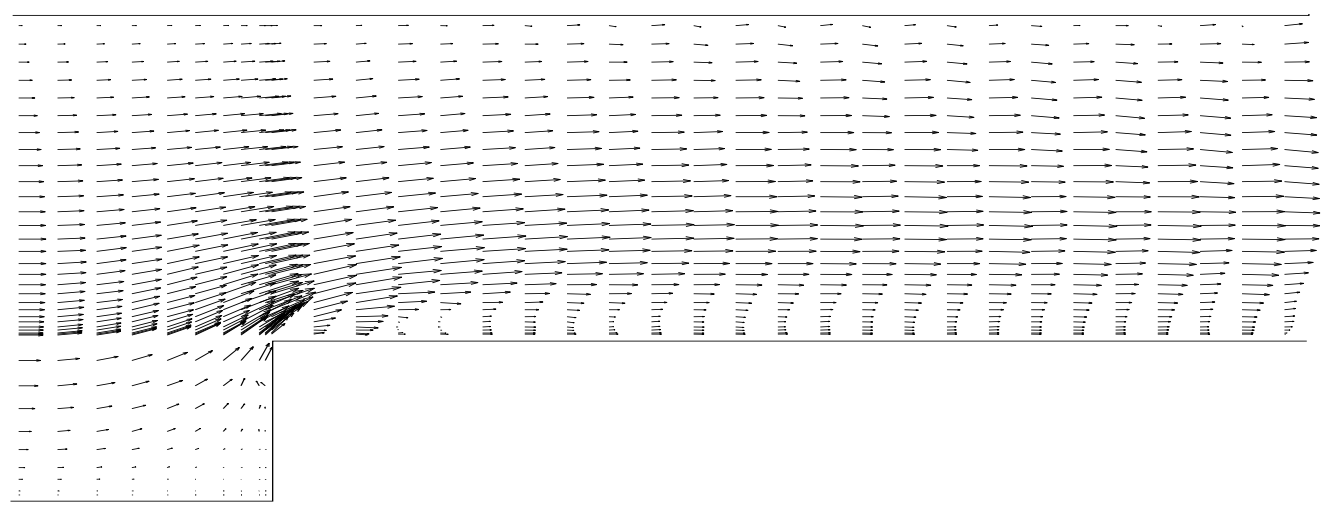

FIG. 19. Controlled channel flow at $\operatorname{Re}=1000$

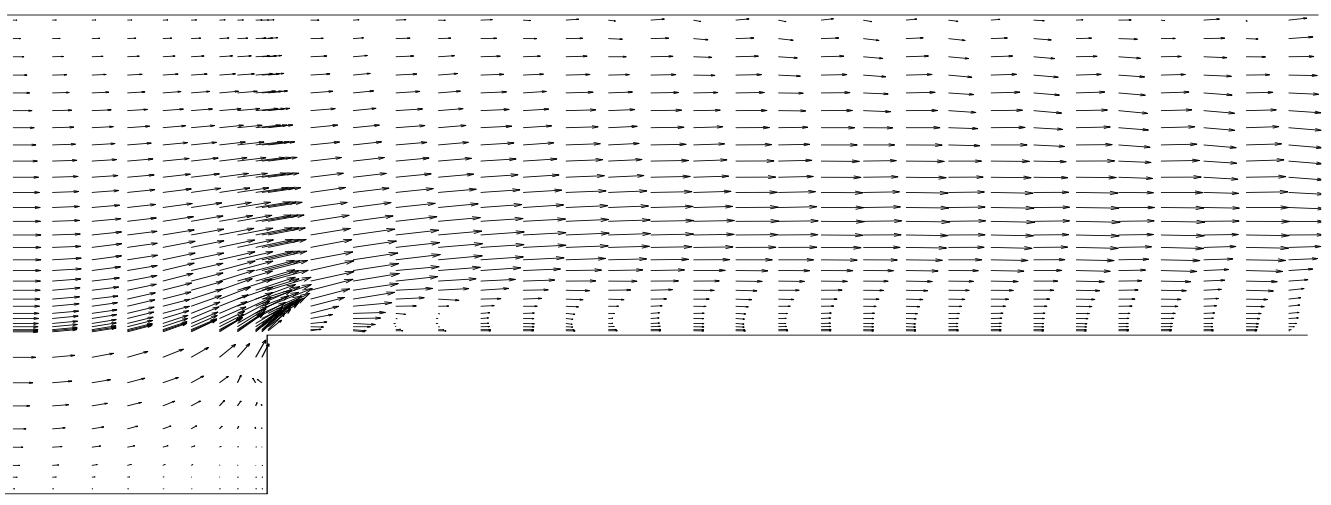

FIG. 20. Channel flow with optimal control input at $R e=1000$ 